\title{
Phase Transition in a Non-Cooperative Population Game*
}

\author{
Heron Carlos Esvael do Carmo* \\ Gustavo Gomes de Freitas***
}

\begin{abstract}
We analyze the random field model applied to a non-cooperative social game of binary choices, as proposed by Durlauf (1997) and Brock and Durlauf (2001), but we allow for a more generic distribution of the agents' heterogeneity.

We aim to show that their results still hold, i.e., there can be multiple equilibria when agents have some interest in adjusting their strategic behavior to that prevailing as the aggregate choice and we discuss some features such as positive feedbacks, the social multiplier, and the possibility of increasing returns on the outcomes of economic policy due to social interactions.
\end{abstract}

Keywords: Complexity, Social Multiplier, Lock-In, Increasing Returns, Diffusion of Innovations.

JEL Codes: C72, D01.

${ }^{*}$ Submitted in February 2006. Revised in June 2007. The authors are grateful to Décio Kadota, Eleutério Prado, Jorge Soromenho, Jaylson da Silveira, Gilberto Lima, Fernando Postali, and José Albertone Pinho for their suggestions, and to two anonymous referees, for their careful observations. Any remaining errors are the sole responsibility of the authors.

${ }^{* *}$ IPE/FEA/USP, Av. Prof. Luciano Gualberto, 908, FEA 2, CEP 05508-010, São Paulo SP. E-mail: hcec@usp.br

*** CCSA/Mackenzie, Rua Itambé, 45, Prédio T, CEP 01239-001, São Paulo, - SP e Grupo Complex - IPE/USP. E-mail: ggfreitas@yahoo.com

Brazilian Review of Econometrics v. $27, \mathrm{n}^{\circ} 2$, pp. $235-259 \quad$ November 2007 


\section{Introduction}

Economists and interdisciplinary research centers have increasingly used modeling techniques related to the so-called Complex Systems for the study of some issues of long-standing interest in Economic Science.

This literature has aroused the interest of renowned economists (Arrow (1994); Kirman (1997); Colander (2000)) for allowing the formal treatment of some issues of continued interest in Economic Science; however, there is still no agreement among scientists with regard to what should be understood under Complex System, even when applied to Social Sciences. ${ }^{1}$

For instance, some works utilize techniques originally used in Statistical Physics: Foley (1994) developed a model of statistical equilibrium for transactions among economic agents, reconsidering the results related to the Walrasian equilibrium and its Pareto efficiency; Blume $(1993,1997)$ modeled the strategic behavior of economic agents in population games; Glaeser et al. (1996) worked with a model of spatial contagion to analyze the geographical diffusion of crime; Topa (2001) used a similar technique to take into account the effect of "word of mouth" dissemination of information for the spatial formation of unemployment rate patterns; and Arthur (1994) presented models of stochastic dynamics to assess technological innovation processes and the chances of survival of inferior technologies, in agreement with the historical evidence amassed by David (1985).

While working with the evolutionary game theory, originally used in Mathematical Biology (Samuelson (1996); Weilbull (1997)), Kandori et al. (1993) showed that the likelihood of mutations in the strategic behavior of a population of players, throughout time, reduces the number of possible equilibria in the game, thus contributing towards equilibrium selection; and Young (1993) analyzed how the traditional social behavior of economic agents may result in a dynamic process in which successive populations of players play the same social game and where the successor populations take good advantage of the fitness data concerning the different strategies utilized by predecessor populations. ${ }^{2}$

By also using the evolutionary game theory, Chiappin (1997) simulated iterative processes for the dynamics of a social game in which agents' behaviors are conditional on political and social institutions; Silveira (2001) developed agent modeling using limited rationality and discussed the competitive process and the development of Goodwinian models of economic growth; Prado et al. (2003) analyzed the conditions under which different technologies survive in the dynamics of market competition; and Prado and Belitsky (2003) used techniques usually applied to particle systems to discuss the possibility of speculative bubbles in financial markets.

\footnotetext{
${ }^{1}$ Among the several studies on the relationship between Economics and Complexity, we recommend Arthur et al. (1997), Arthur (1999), Auyang (1999), and Guedes (1999).

${ }^{2}$ Detailed reviews of this literature are Kirman (1997), Brock and Durlauf (2000), and Glaeser and Scheinkman (2001).
} 
The present paper focuses on the random field model (Kindermann and Snell (1980); Karr (1990)) suggested by Durlauf (1997) and Brock and Durlauf (2001) in order to analyze the existence and the multiplicity of equilibria in a noncooperative social game. However, unlike those authors, who deal with the special case of logistic distribution for agents' heterogeneity, we seek to generalize the results for equilibria by using a general distribution $F(x)$ for such heterogeneity, thus extending the analysis proposed by Glaeser and Scheinkman (2001).

The present paper also aims at shedding further light on the similarity between the adaptation of this technique for use of the model in economics, as suggested by those authors, and the structure of the individual choice model with random utility, which has been well described in the economic literature (McFadden (1981), Griffiths et al. (1993), Greene (2000)).

This is a game in which heterogeneous economic agents make binary choices. Their respective endogenously determined pay-offs depend on the importance given to their expectations as to which behavior will prevail in society vis-à-vis their individual interests. If little or no importance is given to the behavior expected to prevail in society, the equilibrium exists and it is unique; on the other hand, if these same agents give importance to the behavior expected from society by choosing according to the strategy that prevails in the aggregate, there will be multiple equilibria, two of which are socially inferior.

If, for example, agents make a non-cooperative decision to adopt a new technology, the greater importance they attribute to their expectations about the likely social behavior may stem from network externalities; and the two socially inferior equilibria therefore represent a situation in which society finds itself locked into an inefficient configuration, due to the prevalence of the technically inferior alternative.

Moreover, if there is a possibility of replacing multiplicity with uniqueness of equilibria, the economic agents of this society may show a "herd behavior", and therefore, the concept of Social Multiplier will be present, which is understood as the process whereby a small change in the economic agents' individual incentives results in significant changes to society's aggregate behavior (Schelling (1973); Glaeser and Scheinkman (2001)). This phenomenon is also known as phase transition.

The present paper is organized as follows: initially, we analyze the basic structure of the individual choice model with random utility. Then, we introduce the concept of social neighborhood and use the framework of random field models to assess the aggregate behavior of a population of agents whose choices are conditional on social and institutional motivations. Afterwards, we discuss the existence of multiple equilibria for aggregate behavior, as well as the role of social motivations so that we can observe some of the features of population behavior, i.e., emerging patterns, lock-in, increasing returns, and phase transition. Finally, we analyze some implications of these features on the effect of economic policy. 


\section{The Group: Heterogeneous Agents Without Social Interactions}

\subsection{Individual decisions}

As proposed by Durlauf (1997) and Brock and Durlauf (2001), consider a decision problem faced by agent $i$ when choosing between two alternatives, generically represented herein by 0 and 1 .

Let $V_{i}\left(w_{i}\right):\{0,1\} \rightarrow \mathfrak{R}$ be a random utility function that can be decomposed into two additive terms, $u\left(w_{i}\right)$ and $\epsilon_{i}\left(w_{i}\right)$, the deterministic and random terms, respectively, such that

$$
V_{i}\left(w_{i}\right)=u\left(w_{i}\right)+\epsilon_{i}\left(w_{i}\right)
$$

Term $u\left(w_{i}\right)$ denotes the identifiable motivations of agent $i$ 's behavior when choosing $w_{i}$.

If, for instance, choice $w_{i}=1$ refers to the adoption of a new technology by agent $i$, this term $u\left(w_{i}\right)$ may represent the technical gain in an objective costbenefit analysis, obtained by individual $i$ when deciding for the new technology and, which, for being technical, does not depend on his personal features.

The second term in expression $(1), \epsilon_{i}\left(w_{i}\right)$, refers to the agent's latent, unobserved and idiosyncratic motivations in relation to choice $w_{i}$, which are ultimately responsible for the probabilistic feature of his choice behavior.

In the example of adoption of a new technology, this stochastic term may refer to the gain agent $i$ perceives from his individual relationship with innovation, comparatively to the one perceived from the use of old technology, and which, therefore, is essentially personal and depends on his skills.

Given this unpredictable behavior and as a hypothesis of construction, the model assumes that

$$
\operatorname{Prob}\left(w_{i}=1\right)=\operatorname{Prob}\left(V\left(w_{i}=1\right)>V\left(w_{i}=0\right)\right)
$$

i.e., the greater the probability that $w_{i}=1$ yields a higher utility than $w_{i}=0$, the greater the probability that the former will be chosen instead of the latter.

By substituting expression (1) into (2), after some algebraic manipulations, we have

$$
\operatorname{Prob}\left(w_{i}=1\right)=\operatorname{Prob}\left(\epsilon_{i}\left(w_{i}=1\right)-\epsilon_{i}\left(w_{i}=0\right)>u\left(w_{i}=0\right)-u\left(w_{i}=1\right)\right)
$$

or, by inverting the inequality in brackets, we have the following expression 


$$
\operatorname{Prob}\left(w_{i}=1\right)=\operatorname{Prob}\left(\epsilon_{i}\left(w_{i}=0\right)-\epsilon_{i}\left(w_{i}=1\right)<u\left(w_{i}=1\right)-u\left(w_{i}=0\right)\right)
$$

for the probability that choice $w_{i}=1$ will hold. ${ }^{3}$

Now, considering term $\epsilon_{i}\left(w_{i}=0\right)-\epsilon_{i}\left(w_{i}=1\right)$, which is a consequence of the intrinsic heterogeneity of the population, due to the diversity of unobserved individual motivations, which vary from individual to individual, let its behavior be like that of a random variable, which requires an appropriate probability distribution. ${ }^{4}$

Thus, for this random variable $X=\epsilon\left(w_{i}=0\right)-\epsilon\left(w_{i}=1\right)$, whose behavior denotes the agents' heterogeneity, let us define its sampling space as $\Omega=(-\infty, \infty)$, and $f(x)$ and $F(x)$ as the probability density function and accumulated distribution function, respectively. The latter is interpreted as follows: $F(a)$ is equal to the probability of $X$ being smaller than $a$, i.e., $F(a)=\operatorname{Prob}(X<a)$.

After the introduction of these concepts, expression (4) then becomes

$$
\operatorname{Prob}\left(w_{i}=1\right)=\operatorname{Prob}\left(X<u\left(w_{i}=1\right)-u\left(w_{i}=0\right)\right)=F\left(u\left(w_{i}=1\right)-u\left(w_{i}=0\right)\right)
$$

which is the same as to say that the probability of choice $w_{i}=1$ by agent $i$ is equal to the probability of unobserved net incentives in choice $w_{i}=0$ being smaller than the observed net incentives in $w_{i}=1$.

Expression (5) shows that, as a consequence of the definition of $F(x)$ as accumulated probability distribution, and since this is not a decreasing function, the greater the explicit incentives for choosing $w_{i}=1$ when compared to those for choosing $w_{i}=0$, the smaller the uncertainty over the agent's likely behavior, with the latent and unobserved incentives losing dominance over the choice process, since the greater the difference $u\left(w_{i}=1\right)-u\left(w_{i}=0\right)$, the less likely variable $X$ will exceed it.

\subsection{From individual decisions to aggregate behavior}

By extending the analysis, let us consider now not one, but $I$ individuals, each of them having to simultaneously and independently choose $w_{i} \in\{0,1\}$.

Given the decision of each of these agents $I$, the joint behavior of this group is represented by vector

\footnotetext{
${ }^{3}$ With the purpose of presenting an intuitive meaning for expression (4), suppose that a given agent $i$ opted for $w_{i}=0$, although his observed incentives were such that $u\left(w_{i}=1\right)>u\left(w_{i}=0\right)$, i.e., that the choice $w_{i}=1$ appeared to be the rational one, in net terms; therefore, one infers that his implicit and unobserved incentives, also in net terms, i.e., $\epsilon_{i}\left(w_{i}=0\right)-\epsilon_{i}\left(w_{i}=1\right)$, prompted him more strongly to choose $w_{i}=0$, exceeding the observed net incentives, $u\left(w_{i}=1\right)-u\left(w_{i}=0\right)$ for $w_{i}=1$. For a formal analysis of stochastic revealed preference, refer to McFadden (op cit, p. 204).

${ }^{4}$ The features of this probability distribution will be discussed further ahead in this paper.
} 


$$
\mathbf{w}=\left(w_{1}, w_{2}, w_{3}, \ldots, w_{I-1}, w_{I}\right) \in\{0,1\}^{I}
$$

By initially assuming that each of them makes his choice independently from the others, i.e., that choices $w_{i}$ are independent random variables, this vector $w$ might be treated as a random variable whose probability distribution equals the product of the probability distributions of the individual behaviors of each of the agents $I$. Moreover, and also as a consequence of the hypothesis of independent choices (or random experiments, from the observer's point of view), the aggregate behavior of this group of individuals, represented by

$$
m=\left(\Sigma_{i} w_{i}\right) / I
$$

corresponds to the frequency $m$ of choices $w_{i}=1$ in $I$ Bernoulli trials, which has a binomial distribution and, therefore, the expected aggregate behavior of society becomes:

$$
m^{*}=E(m)=F\left(u\left(w_{i}=1\right)-u\left(w_{i}=0\right)\right)
$$

\subsection{The aggregate behavior}

However, let us now assume that the set of agents $I$ constitutes not only a group of individuals, but a society indeed in the sense that there is some kind of behavioral interaction among them and, therefore, some kind of correlation or, more precisely, of dependence in their choice decisions.

Probabilistically, this means that the random variables $w_{i}$ 's that constitute vector $\mathbf{w}$ are no longer independent trials, and that agent $i$ may influence (and also be influenced by) the choice of agent $j, w_{j}$ which may influence (and also be influenced by) the choice of agent $k, w_{k}$, and so on and so forth, for all agents $I$, thus invalidating result (8) for aggregate behavior $\mathrm{m}^{*}$, based on the hypothesis of independence of realizations or choices $w_{i}$ 's.

By analyzing the choice of $I$ individuals who, besides being agents, are also interagents, the model developed so far must be improved, requiring adequate treatment of the interdependence of the random variables $w_{i}$ 's that form society's descriptive vector $\mathbf{w}$.

\section{The Society: Heterogeneous Agents with Social Interactions}

\subsection{The individual choice model with social interactions}

Going back to the problem with the choice of individuals, let $\{-1,1\}$ denote the set of alternative options available to each of the $I$ agents in this society. ${ }^{5}$

\footnotetext{
${ }^{5}$ The change in the representation of the set of choices from $\{0,1\}$ to $\{-1,1\}$ is a mere algebraic tool aimed to facilitate the future development of the analysis.
} 
In a direct analogy or generalization of the analysis previously conducted for the case of independent agents, let us assume now that individual $i$ in his decision process for $w_{i} \in\{-1,1\}$, in addition to the explicit and observed incentives, represented by $u\left(w_{i}\right)$, and to the implicit and idiosyncratic incentives, represented by $\epsilon_{i}\left(w_{i}\right)$, may also consider some kind of social motivation, in such a way that the choices and behaviors of the remaining agents who serve as reference for him, i.e., his social neighborhood, may have some influence on his choice. ${ }^{6}$

As previously mentioned, with regard to agent $i$ 's decision for a new technology, besides the objective motivations concerning the technical efficiency of this new option in relation to the previous ones, the individual may consider incentives related to network externalities. If, for example, positive externality allows easily obtaining information on resources and features made available by such technology from neighbors who have adopted the same technology, agent $i$ will have an additional, contextual and neighborhood-dependent incentive. ${ }^{7}$

Let Neighborhood of individual $i$, denoted by $n(i)$, be the set of agents of society with whom he interacts and who influence his behavior.

And, for simplicity of notation, let $n(i)$ represent the number of agents in agent $i$ 's neighborhood. ${ }^{8}$

Let the additive term $S_{i}\left(w_{i}, \mathbf{w}^{e}\right)$ in $V_{i}\left(w_{i}\right)$ represent the social motivation of agent $i$ 's choice, as a result of the behavior of his neighbors $j \in n(i)$, where $\mathbf{w}^{e}$ corresponds to the expectation that this agent $i$ has in relation to the choice of all the other individuals inserted in his social neighborhood who exert some influence on him.

The inclusion of this social term $S_{i}\left(w_{i}, \mathbf{w}^{e}\right)$ in the random utility function $V_{i}\left(w_{i} ; \mathbf{w}^{e}\right):\{-1,1\} \rightarrow \mathfrak{R}$ changes it to

$$
V_{i}\left(w_{i} ; \mathbf{w}^{e}\right)=u\left(w_{i}\right)+S_{i}\left(w_{i}, \mathbf{w}^{e}\right)+\epsilon_{i}\left(w_{i}\right)
$$

and may be interpreted as the inclusion of a new "variable", actually a function, resulting from the effort towards trying to reduce the model's error or the importance of the random term influencing the agents' choices. Thus, this technique seeks to take into account a motivation for the agent's behavior not considered until then and, therefore, embedded in implicit and unobserved motivations. ${ }^{9}$

Given this extension of the model, still under the same assumption that the individual will be more prone to choose $\left(w_{i}\right)$, the greater the return he expects to

\footnotetext{
${ }^{6}$ Since this is a probabilistic model, again we do not state that the neighborhood has an influence on the agent's choice, but rather that it may have an influence on it.

${ }^{7}$ It seems also possible to assume that these social motivations result from the existence of informal institutions in society, which do not necessarily determine but can condition the agents' individual behaviors and, consequently, the behavior of society (Olson (1971); North (1990)).

${ }^{8}$ The context in which $n(i)$ is inserted makes it clear when either one or the other meaning is intended, with no risk of misinterpretation.

${ }^{9}$ In econometric terms, this would be the same as eliminating error autocorrelation by the inclusion of social determinants, until then regarded as "omitted variables".
} 
obtain by choosing it - when compared to alternative $\left(-w_{i}\right)$ - the probability of agent $i$ choosing $w_{i}$, which depends on the behavior he expects to observe in his neighborhood, will be

$$
\operatorname{Prob}\left(w_{i} \mid \mathbf{w}^{e}\right)=\operatorname{Prob}\left(V_{i}\left(w_{i} ; \mathbf{w}^{e}\right)-V_{i}\left(-w_{i} ; \mathbf{w}^{e}\right)>0\right)
$$

which, by using expression (9), may be rewritten as

$$
\begin{aligned}
\operatorname{Prob}\left(\mathbf{w}_{i} \mid w^{e}\right) & = \\
\operatorname{Prob}\left(\left(\epsilon_{i}\left(-w_{i}\right)\right.\right. & \left.\left.-\epsilon_{i}\left(w_{i}\right)\right)<\left(u\left(w_{i}\right)-u\left(-w_{i}\right)\right)+\left(S_{i}\left(w_{i}, \mathbf{w}^{e}\right)-S_{i}\left(-w_{i}, \mathbf{w}^{e}\right)\right)\right)
\end{aligned}
$$

and, by analogy with expression (5), we have

$$
\operatorname{Prob}\left(w_{i} \mid \mathbf{w}^{e}\right)=F\left(\left(u\left(w_{i}\right)-u\left(-w_{i}\right)\right)+\left(S_{i}\left(w_{i}, \mathbf{w}^{e}\right)-S_{i}\left(-w_{i}, \mathbf{w}^{e}\right)\right)\right)
$$

for the probability of the $i$-th agent choosing $w_{i}$.

\subsection{Specifying the social interaction structure}

Let parameter $J_{i j}$ be a measure of the importance attributed by agent $i$ to the choice of his neighbor $j$ and consider expression

$$
\left(w_{i}+w_{j}^{e}\right)^{2}
$$

with the following property:

A. if agent $i$ 's choice differs from the choice he expected to observe in agent $j$, one will be the opposite of the other, $w_{i}=-w_{j}^{e}$, or still, $w_{i}+w_{j}^{e}=0$ and, finally, $\left(w_{i}+w_{j}^{e}\right)^{2}=0$

B. if, alternatively, agent $i$ 's choice coincides with the choice he expects to observe in agent $j$, then $w_{i}=w_{j}^{e}$ and, or $w_{i}+w_{j}^{e}=+2 o r w_{i}+w_{j}^{e}=-2$ and, thus, $\left(w_{i}+w_{j}^{e}\right)^{2}=4$.

In this case, if we denote the extent of social interaction of individual $i$ with his neighborhood by the expression

$$
S_{i}\left(w_{i}, \mathbf{w}^{e}\right)=\Sigma_{j \in n(i)} J_{i j}\left(w_{i}+w_{j}^{e}\right)^{2}
$$

we will have, with some algebraic treatment combined with the previously described properties of $\left(\mathbf{w}_{i}+w_{j}^{e}\right)^{2}$, the simplified expression

$$
S_{i}\left(w_{i}, \mathbf{w}^{e}\right)-S_{i}\left(-w_{i}, \mathbf{w}^{e}\right)=\Sigma_{j \in n(i)} 4 J_{i j} w_{i} w_{j}^{e}
$$


This expression of social incentive difference according to agent $i$ 's choices may be interpreted as follows: if $J_{i j}>0$, i.e., if agent $j$ 's behavior is positively considered by agent $i$, there is a social incentive for the latter to mimic the behavior of the former, $w_{i}=w_{j}^{e}$, because, in this case, $J_{i j} w_{i} w_{j}^{e}=J_{i j}>0$ and, given the choices of all his remaining neighbors, $S_{i}\left(w_{i}, \mathbf{w}^{e}\right)-S_{i}\left(-w_{i}, \mathbf{w}^{e}\right)>0$. In other words, when $J_{i j}>0$, there is a social incentive for agent $i$ to conform to or mimic the behavior expected for agent $j$.

On the other hand, the opposite is expected when $J_{i j}<0$, i.e., individual $i$ has some social incentive to differentiate his behavior from that of agent $j$, because when $w \neq w_{j}^{e}$, then $J_{i j} w_{i} w_{j}^{e}=-J_{i j}>0$, and, again, if the choices of his remaining neighbors are made, $S_{i}\left(w_{i}, \mathbf{w}^{e}\right)-S_{i}\left(-w_{i}, \mathbf{w}^{e}\right)>0$.

The adoption of a parametric representation $u\left(w_{i}\right)=h w_{i}+k$ for the observed individual incentives with the aim of improving the model in analytical terms leads to the following simplification

$$
u\left(w_{i}\right)-u\left(-w_{i}\right)=\left(h w_{i}+k\right)-\left(h\left(-w_{i}\right)+k\right)=2 h w_{i}
$$

in such a way that the larger (positive) parameter $h$, the larger the observed incentives in dmfavor of $w_{i}=1$ and, consequently, the greater the probability of making the latter choice.

Finally, by using expression (12) for the probability of individual $i$ choosing $w_{i}$ vis-à-vis $-w_{i}$, and given expressions (15) and (16), we have

$$
\operatorname{Prob}\left(w_{i} \mid \mathbf{w}^{e}\right)=F\left(2 h w_{i}+\Sigma_{j \in n(i)} 4 J_{i j} w_{i} w_{j}^{e}\right)
$$

for the probability of agent $i$ choosing behavior $w_{i}$ depending on what he expects to observe in his neighborhood.

Let us now look at the special case where the intensity of interaction among agents is a feature of neighborhood $n(i)$ and not particular to each pair of individuals $i$ and $j$. That is, let us consider that the neighborhood can be characterized by a parameter $J$, in such a way that the intensity of interaction between an individual $i$ and each of the remaining agents $j$ in this neighborhood becomes

$$
J_{i j}=J / 2 n(i), \forall j \in n(i)
$$

The definition of this intensity of interaction between $i$ and $j$ implies that the importance given by agent $i$ to agent's $j$ choice is lower when the former includes more agents in his neighborhood, i.e., the magnitude of interaction $J_{i j}$ is inversely proportional to the size of the neighborhood $n(i)$ of this $i$-th agent. ${ }^{10}$

In this case, the substitution of expression (18) into (17) leads to

$$
\operatorname{Prob}\left(w_{i} \mid \mathbf{w}^{e}\right)=F\left(2\left(h+J \Sigma_{j \in n(i)} w_{j}^{e} / n(i)\right) w_{i}\right)
$$

\footnotetext{
${ }^{10}$ Number 2 in the denominator is a mere mathematical tool used for the mathematical simplification of subsequent expressions.
} 
Finally, if analogously to the aggregate behavior of society, $m$, one defines measure

$$
m_{n(i)}^{e}=\Sigma_{j \in n(i)} w_{j}^{e} / n(i)
$$

as the aggregate behavior that agent $i$ expects to observe in his neighborhood $n(i)$, we will have

$$
\operatorname{Prob}\left(w_{i} \mid m_{n(i)}^{e}\right)=F\left(2\left(h+J m_{n(i)}^{e}\right) w_{i}\right)
$$

for the probability of agent $i$ choosing option $w_{i}$, conditional on his expectation $m_{n(i)}^{e}$ concerning the likely aggregate behavior of his neighborhood.

\subsection{A preliminary analysis of the influence of social incentives}

A feature of this model is that increases in interaction parameter $J$ result in a greater probability of agent $i$ conforming his behavior to the average behavior expected from the group, $m_{n(i)}^{e}$. This happens because by using expression (21), we have

$$
\partial \operatorname{Prob}\left(w_{i}\right) / \partial J=2\left(m_{n(i)}^{e} w_{i}\right) f\left(2\left(h+J m_{n(i)}^{e}\right) w_{i}\right)
$$

and because $f\left({ }^{*}\right)>0$, by its own definition, the sign of this expression will depend exclusively on the product $\left(m_{n(i)}^{e} w_{i}\right)$. Therefore, expression $(22)$ will be positive when $m_{n(i)}^{e}$ and $w_{i}$ have the same sign, which means an increase in the probability of individual $i$ conforming his choice $w_{i}$ to the choice made by most of his neighbors, given by the sign of $m_{n(i)}^{e}$.

\section{The Social Game as a Special Case}

Let us now consider the situation of a social game in which agent $i$ gives importance to the behavior of the whole society, i.e., the aggregate behavior of society is relevant for his choice. In this case, $w_{i} \in\{-1,1\}$ will be understood as the set of game strategies available to each player $i \in\{1,2,3, \ldots, I-1, I\}, V_{i}\left(w_{i} \mid m^{e}\right)$ will be the random pay-off, whose maximization should - probably - guide the strategic behavior, and expression

$$
\operatorname{Prob}\left(w_{i} \mid m^{e}\right)=F\left(2\left(h+J m^{e}\right) w_{i}\right)
$$

is understood as the probability of $w_{i}$ being the strategic behavior of the $i$-th player.

\subsection{The configuration of social equilibrium}

Equilibrium is understood herein as the macroconfiguration $m^{*}$ in such a way that, in terms of aggregate behavior, it simultaneously corresponds to the expectations $m^{* e}$ of all agents at the micro level, discouraging any change in their 
individual decisions. Thus, if these decisions remain unchanged - or more precisely, do not vary much - the aggregate behavior will be maintained as such and will constitute a kind of social equilibrium, a configuration in which society remains, an agreement between the motivations of individuals and the aggregate state resulting from them.

Note that it is not necessary that the agents make their individual choices so that there is an aggregate equilibrium, i.e., it is possible to achieve an equilibrium macrostate for society, although individuals continuously modify their choices at the microeconomic level.

Thus, if we imagine society to be divided into two large groups, more precisely the group of agents that choose $w_{i}=1$ and the group of agents that choose $w_{i}=-1$, the configuration of aggregate social equilibrium can be understood as resulting from the stability in the migratory flows between these two groups, in such a way that the number of agents that leave the first group and enter the second group is equal to the number of agents that leave the second group and enter the first one. Then, although total stability does not exist at the micro level, there is stability at the macro level, which is known as Emerging Pattern in the literature on complex systems.

Since this is a probabilistic system that contemplates the heterogeneity of individual decisions, the concept of equilibrium is understood as a mean value. ${ }^{11}$

This equilibrium is reached when for every agent $I$

$$
E\left(w_{i} \mid m^{*}\right)=m^{*}
$$

i.e., the expected individual choice, for each agent $i, E\left(w_{i} \mid m^{*}\right)$, corresponds to the mean choice observed in the aggregate, $m^{*}$.

When calculating the mathematical expectation of the 1 st item of this equilibrium condition

$$
m^{*}=E\left(w_{i} \mid m^{*}\right)=\left(w_{i}\right) \operatorname{Prob}\left(w_{i} \mid m^{*}\right)+\left(-w_{i}\right) \operatorname{Prob}\left(-w_{i} \mid m^{*}\right)
$$

and considering that

$$
\operatorname{Prob}\left(w_{i} \mid m^{*}\right)+\operatorname{Prob}\left(-w_{i} \mid m^{*}\right)=1
$$

expression (25) may undergo the following algebraic manipulation:

$$
m^{*}=2 w_{i} \operatorname{Prob}\left(w_{i} \mid m^{*}\right)-w_{i}
$$

and then, taking $w_{i}=1$, given expression (23), we will have

\footnotetext{
${ }^{11}$ In other words, this statistical equilibrium describes the behavior of a "mass" phenomenon, i.e., a joint phenomenon of many components or a problem of many bodies. In the literature on Random Fields, these equilibria are known as Mean Field Equilibria because the origins of the models are in the Physics of Ferromagnetism, i.e., in the property of spontaneous magnetization shown by some materials.
} 


$$
m^{*}=2 F\left(2\left(h+J m^{*}\right)\right)-1
$$

as the final expression for the analysis of whether multiple equilibria exist.

\subsection{Existence and multiplicity of social equilibria}

Now, under this condition, it is interesting to analyze whether there is some aggregate configuration $m^{*}$ for social equilibrium or stability and, if there is one, whether this configuration is unique; mathematically speaking, this is the same as to verify, respectively, if there is a value $m^{*}$ that satisfies equilibrium equation (28) and if this value is unique.

In this respect, the following Proposition can be made: ${ }^{12}$ If

H.1 $h>0$, i.e., if option $w_{i}=1$ is in objective and measurable terms in a situation of choice without interaction, better than option $w_{i}=-1$;

H.2 J>0, i.e., if agents are somewhat prone to conform their choices to the choice made by most of the society; ${ }^{13}$

H.3 $\forall x, \exists f^{\prime}(x)$, i.e., if the probability density is at least a once-differentiable mathematical function;

H.4 $\forall x, f(-x)=f(x)$, i.e., if the probability density function is symmetrical, which means that if there were no individual incentives for either of the choices $(h=0)$ as well as no social incentive $(J=0)$, the agents, on average, would be indifferent to both, and the expected choice of the population would be $E(m)=0$; and

H.5 if $f(x)$ has only its maximum value in $x=0$ as critical point,

then

T.1 there is at least one mean equilibrium, $m^{*}$;

T.2 if $m^{*}$ is unique, it will have the same sign of $h$; and

T.3 if there are multiple equilibria, there will be at most three equilibria and only one of them will have the same sign of $h$.

\footnotetext{
${ }^{12}$ Brock and Durlauf (2001) present their results concerning the existence and the uniqueness of the equilibrium for the special case in which distribution $f(x)$ is logistic i.e., $f(x)=1 / 1+$ $\exp (-\beta x)$. Glaeser and Scheinkman (2001) present some results for any given distribution $f(x)$. A complementary analysis to these texts is developed here.

${ }^{13}$ The latter type of interaction, in which the agent shows some interest in belonging to the majority may be called Majority Game. In the referred case of technological innovation, if there were a positive externality in its use, we would have a majority game, represented by $J>0$, as in the example of an investor, if his speculative behavior is of the "Beauty Contest" type. (Keynes (1983), Chapter 12).
} 
Proof The argument consists in constructing the function

$$
H(m)=2 F(2(h+J m))-1-m
$$

obtained by the difference between the members of equation (28), showing that there are multiple values for $m$ that could nullify $H(m)$ because, in this case, there are multiple values that satisfy equation (28) and, therefore, there are multiple aggregate configurations of equilibrium for society. ${ }^{14}$

One possibility for the behavior of function $H(m)$ is the one denoted in Figure 1 . This is the case in which social equilibrium $m^{*}$ is unique, since function $H(m)$ crosses the placeOm axis only once. Moreover, since such equilibrium $m^{*}$ is positive and has the same sign of $h$, the majority of society in this aggregate configuration opts for $w i=1$, which is equivalent to the best choice, should just individual motivations be considered.

Unlike the previously illustrated case, however, Figure 2 displays a second possibility, when there are multiple equilibria, since function $\mathrm{H}(\mathrm{m})$ crosses the $\mathrm{Om}$ axis at three different points, i.e. $m_{-}^{*}, m_{0}^{*}$ and $\mathrm{m}^{*}{ }_{+}$, each of them representing an aggregate configuration of social equilibrium. Furthermore, note that in two of these equilibria, specifically $m_{-}^{*}$ and $m *_{0}$ the individual decision of most agents is for the inferior choice $w i=-1$ since $m_{-}^{*}<0$ and $m_{0}^{*}<0$, although $h>0$.

\subsection{Social equilibria as emerging patterns}

The previously described configurations, where $w_{i}=-1$ becomes a majority choice in the aggregate behavior $\left(m_{-}^{*}<0\right.$ and $\left.m_{0}^{*}<0\right)$, are examples of the socalled emerging patterns as found in the literature on Complex Systems, since they can only be explained by the presence of the agents' social motivations: such configurations emerge from the agents' level to the population level not as a mere "sum" of what their individually reasonable choices would be, but as an aggregate "consequence", deriving from the social interaction among the system's agents, represented in the model by parameter $J$.

In order to advance in the analysis with regard to society possibly finding itself in an inferior aggregate configuration $\left(m^{*}<0\right)$, it is important to have a better understanding of the role that the agents' individual incentives plays, denoted by parameter $h$ of the model, vis-à-vis the net social incentives related to interaction parameter $J$.

Let us analyze the situation illustrated in Figure 1: when $h$ is large enough, vis-à-vis $J$, there is only one possible statistical equilibrium $m^{*}$, leaving no doubt about which the society's major strategy will be, i.e., the one that offers the largest individual incentives, which explains why the resulting polarization $m^{*}$ has the same sign of $h$. Also note that the larger these individual incentives are, the

\footnotetext{
${ }^{14}$ For details on the proof, see Appendix A. 
greater the dominance of that strategy, since the higher the $h$ value, the closer $m^{*}$ will be to 1 .

On the other hand, when those same individual incentives vis-à-vis the intensity of interaction among agents are not strong enough - as in the case of Figure 2 - there are three possibilities of equilibrium: $m_{-}^{*}, m_{0}^{*}$ and $m_{+}^{*}$. In this case, the latter denotes the configuration in which the strategy with larger individual incentives will prevail over the agents' choices, and the former two possibilities denote the configurations in which the other strategy will prevail over society's choices.

The possibility of inferior equilibria, in cases where social incentives are very strong - known as lock-in -, is due to the endogeneity of preferences, i.e., agents show interest in conforming their behaviors to the prevailing behavior they expect to see in society. Consequently, social equilibrium may occur either in $m_{-}^{*}$ or in $m_{+}^{*}$ because, in both cases, the agents will not have incentives to change their choices, since they were able to conform them to what they expected society's majority behavior to be, which really matters to them.

Let us take, for example, the case of diffusion of a new behavior in society or even the adoption of a new technology (denoted by $w_{i}=1$ ) that is technically superior to the old technology $\left(w_{i}=-1\right)$ since $h>0$.

If there are social motivations, although moderate, vis-à-vis individual technical gains, the dominance of the new technology will be greater than the one that would occur in the absence of social interactions, which means that more agents opt for the new technology as a consequence of social incentives.

However, if social incentives are too strong, always vis-à-vis individual technical gains, given the desire of each agent to wait for innovation to disseminate in society before opting for a change, the old technology, albeit technically inferior, may continue to prevail for a long time.

This is a case of emerging social behavior because, although the agents are individually favorable to the change $(h>0)$, at the level of aggregation - the social level - and in view of the interactions and the endogeneity of preferences, the agents adopt an "irrational" strategy if their social motivations are not taken into account. However, once these motivations are taken into account, this apparently inexplicable aggregate behavior can be understood, however inefficiently.

\subsection{Comparative static and social multiplier}

The possibility of the superior option not propagating enough to become the society's major choice, in a rushed interpretation, suggests that the problem of society lies in the social characteristic of its agents, which therefore suggests that it is "OK" to subdue it by weakening social ties.

However, a more careful analysis allows us to conclude the following:

A. the agents' social motivations may originate from society's social heritage and, if true, they may not be so easily changed, since this change might require a long process of institutional change; and 
B. social motivations may be occasionally treated as a favorable feature if deliberately and adequately explored. This point of view is justifiable because a temporary incentive program that allows overcoming the critical values that keep it locked in may allow for a chain process of behavioral change vis-à-vis the new technology, resulting from the increasing returns of social interaction and multiplication, in such a way that, after the intervention, society clears away the hurdles and stabilizes in a technically superior aggregate configuration.

An interesting point is to better explore the effect of a variation in parameter $h$ on aggregate equilibrium $m^{*}$.

Graphically, an increase in parameter $h$ may be denoted by upward shifts in each point of the curve $(m, H(m))$, as illustrated in Figures 3 and 4 .

In the first case, where there is only one equilibrium $m^{*}$, this increase in parameter $h$ prompts a greater share of society to opt for strategy $w_{i}=1$, since $m_{2}^{*}>m_{1}^{*}$, i.e., the new equilibrium is larger than the previous one.

In the second case, however, a change in individual incentives can have an even more remarkable effect in the case of 3 equilibria: let us assume that before the variation in parameter $h$, society was in an equilibrium $m_{-}^{*}$, which means that the vast majority of players opted for strategy $w_{i}=-1$. Having overcome the impact of the increase in parameter $h$, depending on the intensity of the change, society is likely to move from multiple equilibria to a unique equilibrium, a change that denotes that the vast majority of players abruptly invert their choices by swapping the previously major option $w_{i}=-1$ for the new dominant option $w_{i}=1$.

This may occur because the change in an agent's choice prompts other agents to change theirs as well, which ends up interfering in the other agents' choices in such a way that the resulting aggregate change is eventually much greater than that resulting from the mere sum of individual changes, should there not be interactions among agents. ${ }^{15}$

Note that in this process of change in equilibrium, the effect of an increase in individual incentives is enhanced by the effect of social incentives, in a process of feedback and increasing returns known as social multiplier.

Mathematically speaking, the effect of the social multiplier process may be analyzed as follows: by taking equation (28), which determines society's aggregate equilibrium $m^{*}$, and by partially differentiating both members in relation to $h$, we have

$$
\partial m^{*} / \partial h=4 f\left(2\left(h+J m^{*}\right)\right) /\left(1-4 J f\left(2\left(h+J m^{*}\right)\right)\right)
$$

\footnotetext{
${ }^{15}$ The change depicted in the previous figure, where the system moves from a major negative aggregate configuration $m_{1}^{*}$ to a major positive aggregate configuration $m_{2}^{*}$, is known as Phase Transition in the literature on Complex Systems. This is closely related to the phase transition shown, for example, by water, which changes from solid to liquid state even if its temperature remains at $0^{\circ} \mathrm{C}$.
} 
an expression that illustrates the role played by parameter $J$ in the existence of a nonlinear effect of a change in parameter $h$ on the configuration of equilibrium $m^{*}$, characteristic of the Social Multiplier concept: the closer the product of $4 J f(2(h+$ $\left.\left.J m^{*}\right)\right)$ ) is to 1 , to which $J$ contributes directly, the closer to zero the denominator of expression (30) will be and, consequently, the greater the aggregate effect of a marginal change in individual incentives. ${ }^{16}$

On the other hand, since

$$
\partial^{2} m^{*} / \partial J \partial h=\left(\partial m^{*} / \partial h\right)^{2}+8 m^{*} f^{\prime}\left(2\left(h+J m^{*}\right)\right) /\left(1-4 J f\left(2\left(h+J m^{*}\right)\right)\right)^{3}
$$

one can evaluate the role played by social interaction parameter $J$ in the presence of a social multiplier. In order to simplify the analysis of this effect, without making it less interesting, let us take a look at the special case in which $\mathrm{m}^{*}=0$, which simplifies expression (31) into

$$
\partial^{2} m^{*} /\left.\partial J \partial h\right|_{m^{*}=0}=\left.\left(\partial m^{*} / \partial h\right)^{2}\right|_{m *=0}
$$

Therefore, in the case of a society whose equilibrium is $m^{*}=0$, since the expression to the right of equation (32) is necessarily positive, the greater the intensity of social interaction among agents, the greater the effect of a change in individual incentives will be on the aggregate behavior, indicating the presence of a social multiplier.

Furthermore, considering the general case $m^{*} \neq 0$, one can obtain the following expression for the effect of a variation in social motivations on the configuration of equilibrium

$$
\partial m^{*} / \partial J=m^{*} \partial m^{*} / \partial h
$$

which highlights two interesting features of the influence of social motivations on society's aggregate behavior:

A. since $\left|m^{*}\right|<1$, we have $\left|\partial m^{*} / \partial J\right|<\left|\partial m^{*} / \partial h\right|$ or, translating it into words, the magnitude of the effect of a small increase in the intensity of social interaction is never larger than the magnitude of the effect of a small increase in individual incentives; and

B. by admitting that an increase in individual incentives increases the number of agents that opt for $w_{i}=1$, i.e., admitting that $\partial m^{*} / \partial h>0$, then, according to expression (33), if $m^{*}>0$, then $\partial m^{*} / \partial J>0$, and if $m^{*}<0$, then $\partial m^{*} / \partial J<0$, which means that society has a self-enforcing mechanism in its aggregate behavior, since an increase in the agents' social incentives increases the dominance of the choice for the prevailing individual behavior.

\footnotetext{
${ }^{16}$ This is true in those cases in which the numerator of expression (30) does not approach zero more rapidly than the denominator does, with an increase of $J$.
} 


\section{Conclusion}

The present paper aimed to analyze the mathematical structure of random field models adapted to some of the interests of Social Sciences.

It dealt particularly with a game in which heterogeneous economic agents make binary choices. Their pay-offs and, consequently, their strategic decisions, depend on the importance they attach to their expectations with regard to the probably prevailing social behavior vis-à-vis their individual interests.

There is always at least one configuration of social equilibrium; however, depending on the importance attributed to the behavior expected from society, choosing according to the strategy prevailing in the aggregate, it is possible to go from single to multiple equilibria, two of which are socially inferior.

Furthermore, the case with multiple equilibria served to illustrate the Social Multiplier.

The analogy of this model with a non-cooperative decision problem in the adoption of a new technology by economic agents was also carried out. The presence and the intensity of network externalities in this economy can lead to a situation in which society sees itself locked into an inefficient configuration, due to behavioral conservatism and a consequent prevalence of the technically inferior option.

The identification of these features in the innovation behavior of societies may be relevant to the discussion on economic policy. A temporary individual incentive program, which triggers a chain process of behavioral change vis-à-vis the new technology and conveniently explores the increasing returns of social interaction and multiplication, may allow society to overcome the conservatism that keeps it locked into inferior aggregate configurations. If the implementation of these incentives succeeds, it may lead society into a phase transition, resulting eventually in a technically superior aggregate configuration.

There are clearly many questions that have not been dealt with herein but that deserve further analysis: a first extension, for example, could be the search for an alternative mathematical treatment of the problem, aiming not only at eliminating the superficialities of the analysis presented but also at revealing other important features of the aggregate behavior which were not identified herein or which were not properly considered.

A second extension could consist of a stochastic evolutionary game approach to analyze whether a dynamic choice process, in which the agents periodically update their decisions, might result in the selection of one of the equilibria, occasionally discarding the socially inefficient aggregate configuration. 
It also seems suitable to attempt dealing with the same problem by a computer simulation of artificial societies, which would allow relaxing some of the hypotheses of the model. One of these hypotheses is that which refers to the structure of interaction of each individual with society as a whole, which could be possibly replaced with the endogenous formation of effectively local neighborhoods, although linked to complex network structures that allow strengthening social motivations and their consequences to the aggregate behavior.

\section{References}

Arrow, K. (1994). Foreword. In Increasing Returns and Path Dependence in the Economy. Michigan University Press, Ann Arbor.

Arthur, W. B. (1994). Increasing Returns and Path Dependence in the Economy. Michigan University Press, Ann Arbor.

Arthur, W. B. (1999). Complexity and the economy. Science, 284(107).

Arthur, W. B., Durlauf, S. N., \& Lane, D. A. (1997). The Economy as an Evolving Complex Sistem II, volume 27. Addison-Wesley, Medwood City. Santa Fe Institute in the Sciences of Complexity.

Auyang, S. Y. (1999). Foundations of Complex System Theories in Economics, Evolutionary Biology and Statistical Physics. Cambridge University Press, Cambridge.

Blume, L. (1993). The statistical mechanics of strategic interaction. Games and Economic Behavior, 27:387-426.

Blume, L. (1997). Population games. In Arthur, W. B., Durlauf, S. N., \& Lane, D. A., editors, The Economy as an Evolving Complex System II. AddisonWesley, Medwood City. Santa Fe Institute Studies in the Sciences of Complexity.

Brock, W. \& Durlauf, S. N. (2000). Interactions-based models. NBER Working Paper 258.

Brock, W. \& Durlauf, S. N. (2001). Discrete choice with social interactions. Review of Economic Studies, 68:235-260.

Chiappin, J. R. N. (1997). Racionalidade, Jogos Dinâmicos, Métodos Estocáticos Markovianos e Comportamento Coletivo. PhD thesis, IPE/USP.

Colander, D. (2000). Complexity and the History of Economic Thought-Perspectives on the History of Economic Thought. Taylor \& Francis, New York.

David, P. (1985). Clio and the economics of QWERTY. American Economican Review - Papers and Proceedings, 75:332-337. 
Durlauf, S. (1997). Statistical mechanics approachs to socioeconomic behavior. In Arthur, W. B., Durlauf, S. N., \& Lane, D. A., editors, The Economy as an Evolving Complex System II, volume 27, pages 81-104. Addison-Wesley, Medwood City. Santa Fe Institute Studies in the Sciences of Complexity.

Foley, D. K. (1994). A statistical equilibrium theory of markets. Journal of Economic Theory, 62:321-345.

Glaeser, E. L., Sacerdote, B. I., \& Scheinkman, J. A. (1996). Crime and social interactions. The Quarterly Journal of Economics, 111:507-548.

Glaeser, E. L. \& Scheinkman, J. A. (2001). Non-market interactions. HIER Working Paper 1914.

Greene, W. (2000). Econometric Analysis. Prentice Hall, New Jersey, 4th edition.

Griffiths, W. E., Hill, R. C., \& Judge, G. G. (1993). Learning and Practicing Econometrics. John Wiley and Sons, New York.

Guedes, F. C. (1999). Economia e Complexidade. Almedina, Coimbra.

Kandori, M., Mailath, G. J., \& Rob, R. (1993). Learning, mutation and long run equilibria in games. Econometrica, 61(1):29-56.

Karr, A. F. (1990). Markov processes. In Heyman, D. P. \& Sobel, M. J., editors, Handbooks in Operations Research and Management Science, pages 95-123. North Holland, New York.

Keynes, J. M. (1983). A Teoria Geral do Emprego, dos Juros e da Moeda. Abril Cultural, São Paulo.

Kindermann, R. \& Snell, J. L. (1980). Markov Random Fields and their Applications. American Mathematical Society, Providence.

Kirman, A. P. (1997). The economy as an interactive system. In Arthur, W. B., Durlauf, S. N., \& Lane, D. A., editors, The Economy as an Evolving Complex System II, volume 27, pages 491-531. Addison-Wesley, Medwood City. Santa Fe Institute Studies in the Sciences of Complexity.

McFadden, D. (1981). Econometric models of probabilistic choice. In McFadden, D. \& Manski, C. F., editors, Structural Analysis of Discrete Data with Econometric Applications. MIT Press, Cambridge.

North, D. (1990). Institutions, Institutional Change and Economic Performance. Cambridge University Press, Cambridge.

Olson, M. (1971). The Logic of Collective Action. Harvard University Press, Cambridge. 
Prado, E. F. S., Kadota, D. K., \& Soromenho, J. E. C. (2003). Survival of technologies: An evolutionary game approach. Economia Aplicada, 7(2):249-265.

Prado, F. P. A. \& Belitsky, V. (2003). Multiple market equilibria, bubbles and crashes explained by heterogeneity of fundamental value evaluations and social susceptibilities of interacting agents. Instituto de Matemática e Estatística, mimeo.

Samuelson, L. (1996). Evolutionary Games and Equilibrium Selection. The MIT Press, Cambridge.

Schelling, T. (1973). Hockey helmets, concealed weapons and daylight saving: A study of binary choice with externalities. Journal of Conflict Resolution, $17: 381-428$.

Silveira, J. J. (2001). Ciclos Goodwinianos e o Processo de Concorrência num Ambiente de Racionalidade Limitada - Uma Análise a partir da Teoria de Jogos Evolucionários. PhD thesis, IPE/USP.

Topa, G. (2001). Social interactions, local spillovers and unemployment. Review of Economic Studies, 68:261-295.

Weilbull, J. (1997). Evolutionary Game Theory. The MIT Press, London.

Young, H. P. (1993). The evolution of conventions. Econometrica, 61(1):57-84. 


\section{Appendix A}

\section{The Existence and Multiplicity of Equilibria}

Let

$$
H(m)=2 F(2(h+J m))-(m+1)
$$

be the function that corresponds to the difference between the members of equilibrium equation (28).

By construction, $0<F(x)<1$, which implies that if $m=-1$,

$$
H(m)=2 F(2(h-J))-(-1+1)=2 F(2(h-J))>0
$$

i.e., $H(m)$ is located above the $O m$ axis when $m=-1$.

Analogously, if $m=1$,

$$
H(m)=2 F(2(h+J))-(1+1)=2 F(2(h+J))-2<0
$$

i.e., $H(m)$ is located below the $O m$ axis, if $m=1$.

Then, the combination of (A.2) and (A.3) ensures that function $H(m)$ will change signs at least once on the interval $[-1,1]$. But, since $H(m)$ is continuous, it is not possible for it to do this without crossing the $\mathrm{Om}$ axis, i.e., without nullifying itself at least once. Thus, it has been proven that there is at least one $m^{*}$ that nullifies $H(m)$ and that, by construction, satisfies equilibrium equation (28). This, therefore, ensures that

T.1) there will be at least one equilibrium of aggregate behavior $m^{*}$.

Furthermore, as a consequence of (H.1) and of (H.4) we have $F(2 h)>50 \%$ and, then,

$$
H(0)=2 F(2 h)-1>0
$$

i.e., $H(m)$ has positive intercept on the $O H$ axis.

Thus, (A.3), (A.4) and the continuity of $H(m)$ ensure that it crosses the positive side of the $O x$ axis at least once, i.e.,

T.2) if $m^{*}$ is unique, it will have the same sign of $h$;

Now, by deriving $H(m)$ we have

$$
H^{\prime}(m)=4 J f(2(h+J m))-1
$$

which, when nullified, determines the following condition to be satisfied by its extreme points:

$$
m_{c}=\left(\left(f^{-1}(1 / 4 J)\right) / 2-h\right) / J,
$$

$f^{-1}(1 / 4 J)$ denoting values of $x$ such that $f(x)=1 / 4 J$. 
However, given the symmetry property (H.4) of $f(x)$, there are two values for $x$, a negative and a positive one, for which $f(x)=1 / 4 J$, namely $x_{E}=$ $-\left|f^{-1}(1 / 4 J)\right|$ and $x_{D}=\left|f^{-1}(1 / 4 J)\right|$ and, by correspondence, there are two points of local extreme value for $H(m)$, with abscissas

$$
m_{c D}=\left(\left(\left|f^{-1}(1 / 4 J)\right|\right) / 2-h\right) / J
$$

and

$$
m_{c E}=\left(\left(-\left|f^{-1}(1 / 4 J)\right|\right) / 2-h\right) / J
$$

of which, the latter is necessarily negative. ${ }^{17}$

Taking now the second derivative of $H(m)$,

$$
H^{\prime \prime}(m)=8 J^{2} f^{\prime}(2(h+J m))
$$

and substituting the abscissas of the critical points in this function, given by (A.7) and (A.8), we have

$$
H^{\prime \prime}\left(m_{c D}\right)=8 J^{2} f^{\prime}\left(\left|f^{-1}(1 / 4 J)\right|\right)<0
$$

and

$$
H^{\prime \prime}\left(m_{c E}\right)=8 J^{2} f^{\prime}\left(-\left|f^{-1}(1 / 4 J)\right|\right)>0
$$

indicating that $m_{c D}$ and $m_{c E}$ are, respectively, of local maximum and local minimum.

By equaling the second derivative of $H(m)$ to zero, we verify that by expression (A.9) its inflection point has

$$
m_{\sim}=-h / J
$$

as abscissa, corresponding to the intermediate position between the abscissas $m_{c E}$ and $m_{c D}$, on the $\mathrm{Om}$ axis

Since $H(m)$ must be increasing on the interval $\left[m_{c E}, m_{c D}\right]$ and since $m_{c E}<m_{\sim}$ $<m_{c D}$, we have

$$
H\left(m_{c E}\right)<H\left(m_{\sim}\right)
$$

and, substituting (A.12) into (A.1) yields

\footnotetext{
${ }^{17}$ It is important to note that, if $f(0)$ is the maximum of $f(x)$, for those cases where $f(0)<1 / 4 J$, there will be one or no solution to (A.6) and, therefore, for the existence of two extreme points of $H(m)$, it is necessary that $J>J_{c}=1 / 4 f(0)$; Prado and Belitsky (2003) assessed the possibility of speculative bubbles in the financial market and associated minimum agent heterogeneity with this restriction, which is enough to avoid a herd behavior among investors.
} 


$$
H\left(m_{c E}\right)<h / J
$$

The combination of expressions (A.1) and (A.8) and the convenient cluster of the resulting terms yields the following local minimum value for $H(m)$ :

$$
H\left(m_{c E}\right)=\left\{2 F\left(-\left|f^{-1}(1 / 4 J)\right|\right)+\left(\left|f^{-1}(1 / 4 J)\right|\right) / 2 J-1\right\}+h / J
$$

However, as the term between braces is a function only of $J$, it may be denoted by $\varphi(J)$ and this expression may be rewritten in a more compact form as

$$
H\left(m_{c E}\right)=\varphi(J)+h / J
$$

which leads to the conclusion that $\varphi(J)<0$, given the restriction imposed by inequality (A.14) on the value of $H\left(m_{c E}\right)$.

By the analysis of two typical cases of major interest, we may now conclude that:

A. when $h$ is large enough, vis-à-vis $J$, for the positive term $h / J$ to exceed, in absolute value, the negative term $\varphi(J)$ in expression (A.16), the value of the local minimum $H\left(m_{c E}\right)$ should be positive and, therefore, the point of local minimum of $H(m)$ is located above the $O m$ axis. This is illustrated in Figure 1 in Appendix B, where we then have a unique social equilibrium, with the same sign of $h$; however;

B. when $h$ is not large enough, vis-à-vis $J$, and term $h / J$ does not exceed, in absolute value, the negative term $\varphi(J)$ in expression (A.16), the value of the local minimum $H\left(m_{c E}\right)$ is negative and, in this case, the point of local minimum of $H(m)$ is located below the $O m$ axis. This is illustrated in Figure 2 in Appendix B, in which, therefore,

T.3 if there are multiple equilibria, there will be a maximum of three equilibria and only one with the same sign of $h$ 
Appendix B

Figures

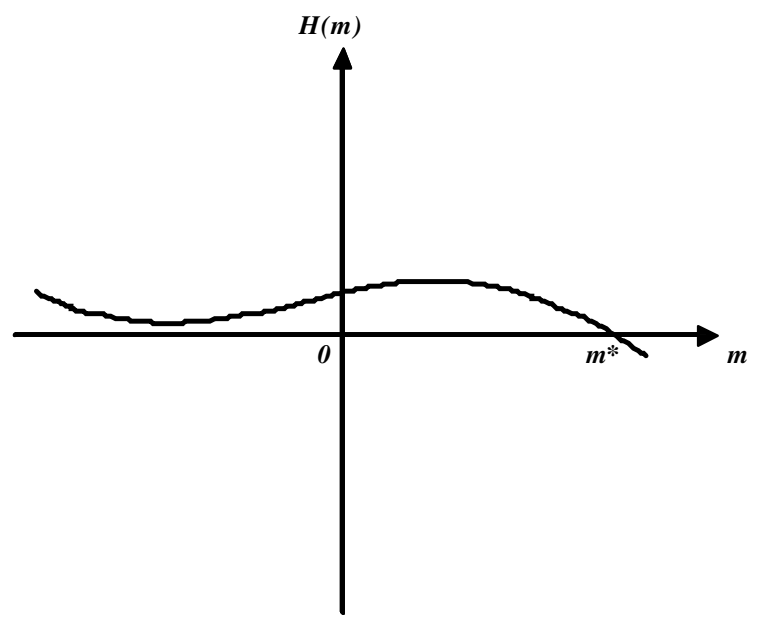

Figure A.1

Unique equilibrium

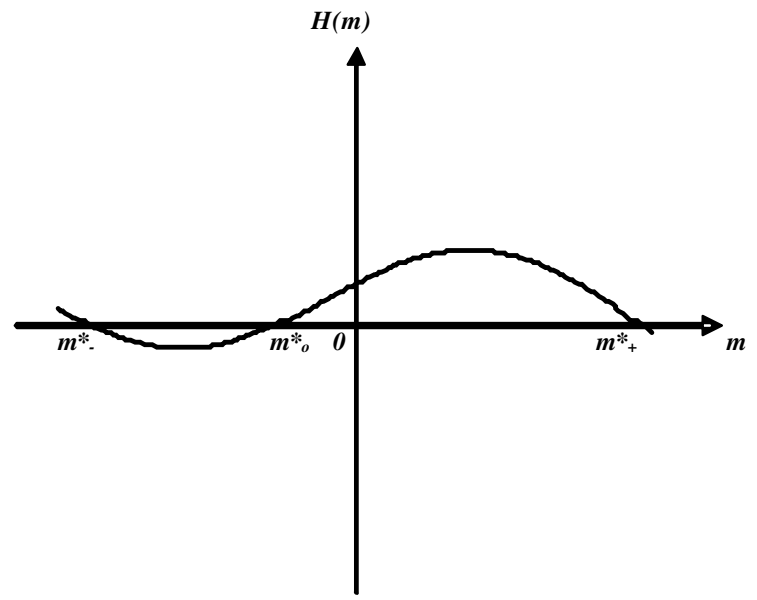

Figure A.2

Multiple equilibria 


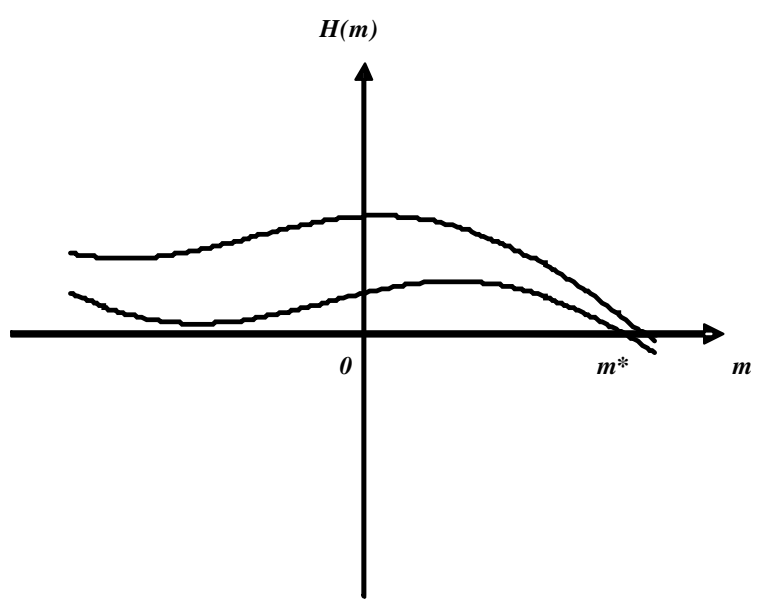

Figure A.3

Uniqueness of equilibrium and incentive effects

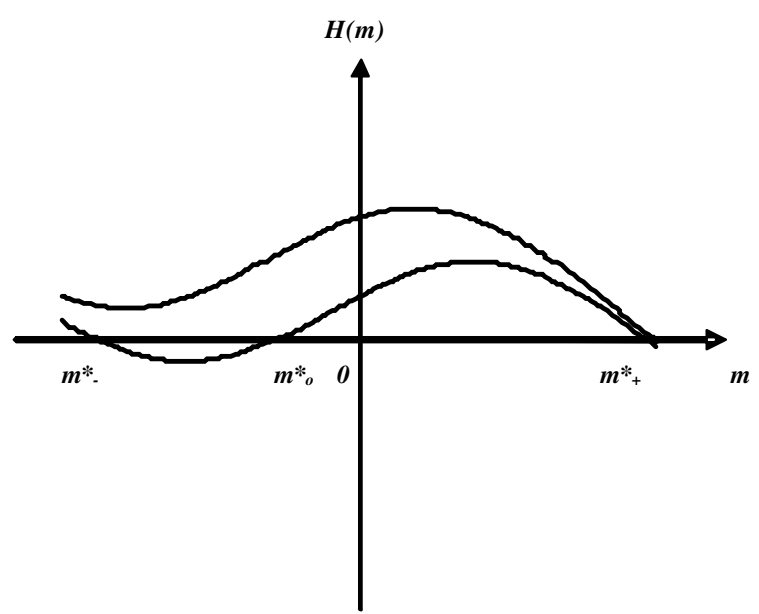

Figure A.4

Multiplicity of equilibria and phase transition 